\title{
Hybrid mesons: old prejudices and new spectroscopy
}

\author{
Yu.S.Kalashnikova ${ }^{a}$ \\ ${ }^{a}$ Institute of Theoretical and Experimental Physics, 117259, Moscow, Russia
}

\begin{abstract}
The models for hybrid mesons are discussed, in which the gluonic excitations manifest themselves as the vibrations of the quark- antiquark QCD string. The predictions for the spectra, decays and mixing with hadronic channels are presented.
\end{abstract}

\section{INTRODUCTION}

The problem of existence or nonexistence of hadrons with gluonic content attracts a lot of attention both from theoretical and experimental point of view. Rather general arguments from QCD tell that the theory, even quenched in quarks, should possess nontrivial spectrum, while the present state-of-art does not allow to derive it from first principles. A quite respectable way to deal with such situation is to try to rewrite the generating functional of QCD in nonperturbative region in terms of constituent degrees of freedom. To formulate a string theory is, apparently, a suitable way to do it.

As the QCD is not a string theory, a question arises: is it possible to introduce the notion of constituent glue in this effective string theory. To answer this question it is instructive to look for experience from more simple theories, namely the two-dimensional QCD in the large $N_{c}$ limit. The $\mathrm{QCD}_{2}$ with fundamental fermions was analysed long ago [1], and it was found that the spectrum of the model is exhausted by $q \bar{q}$ meson states joined by the string. These states lie on the Regge trajectory

$M_{n}^{2}=n g^{2} N_{c}$

with the density of states rising linearly with the mass, $d n / d M \sim M$. The simple spectrum (1) is completely due to the fact that there is no dynamical gluons in the $\mathrm{QCD}_{2}$, and the Coulomb force is confining in two dimensions.

In contrast to t'Hooft model, the $\mathrm{QCD}_{2}$ with fermions which belong to the adjoint representation of the colour group is less trivial even in the large $N_{c}$ limit. It happens because the presence of adjoint matter allows for more complicated string configurations. It was shown by explicit calculations [2] that the number of Regge trajectories is infinite, and the density of states grows exponentially with the mass. Moreover, the very notion of trajectory remains reasonable only for the lowest states; with the increase of the mass more and more states from different trajectories enter the game, the trajectories begin to overlap and the spectrum becomes rather stochastic.

It is the same behaviour as one expects in the $\mathrm{QCD}_{4}$, with dynamical gluons playing the role of adjoint matter. The effective string model should be arranged to allow the gluons to populate the string and to be responsible for complicated string configurations.

\section{SPECTRUM ESTIMATIONS}

The area law asymptotics for the Wilson loop conveniently provides us with the action of the string, and naive expectations from the string spectrum read 
$M^{2}=2 \pi \sigma(L+2 \nu)$

for the string Regge trajectory, were $L$ is the orbital momentum, $\nu$ is the vibrational quantum number, and $\sigma$ is the string tension. Placing quarks at the ends of the string we have new degrees of freedom, so that the Lagrangian of the system becomes

$L=-m_{1} \sqrt{\dot{x}_{1}^{2}}-m_{2} \sqrt{\dot{x}_{2}^{2}}+L_{\text {string }}$.

The system (3) was extensively studied in [3] in the so-called straight-line approximation which corresponds to the special kind of the string motion with frozen vibrations $(\nu=0)$. In the limit of large quark masses the Lagrangian (3) gives rise to the effective quasipotential Hamiltonian for the $q \bar{q}$ system,

$H=\sqrt{p^{2}+m_{1}^{2}}+\sqrt{p^{2}+m_{2}^{2}}+\sigma r$,

and for high excitations the Regge trajectory takes the form

$M^{2}=2 \pi \sigma\left(l+2 n_{r}\right)+$ quark mass corrections. (5)

It is natural to identify the $q \bar{q}$ system connected by straight - line string with conventional $q \bar{q}$ meson, while the string vibrations are responsible for the gluonic excitations of the QCD string, forming hybrids, with Regge trajectory given by

$M^{2}=2 \pi \sigma\left(L+2 n_{r}+2 \nu\right)$.

The lower part of the spectrum (6) is distorted by quark mass corrections, and the actual pattern of distortion depends on the model adopted (see e.g the results from the flux tube model [4] and the constituent string model [5]). It is clear that for hybrids with light quarks there is only one dimensional parameter, $\sqrt{\sigma}$, and, in contrast to nonrelativistic systems, there is no gap between radial/orbital/vibrational excitations in the $q \bar{q}$ system. Quark mass corrections, short range effects and mixing make the trajectories given by oversimplified estimation (5) overlap, and the spectrum is expected to be rather stochastic (like in the $\mathrm{QCD}_{2}$ with adjoint fermions?).

\section{HYBRIDS DECAYS}

The existing models $[4,5]$ agree that the lowest $q \bar{q} g$ hybrids have the masses around $1.7-1.9 \mathrm{Gev}$, ie in the mass region populated by the radial and orbital $q \bar{q}$ mesons. There is also the agreement concerning quantum numbers of the $\mathrm{P}$-odd part of hybrid spectrum:

$J^{P C}=0^{-+}, 1^{-+}, 2^{-+}, 1^{--}$.

It appears that just the same quantum numbers cause a lot of interest in "hybrid hunting".

Indeed, it was established in a lot of ways [6-8] that the famous selection rule exists for hybrid decay: the ground state hybrid does not decay into two ground state mesons. More accurate statement is that the decay matrix element vanishes for two-body final states with identical space wave functions. It means that the main decay modes of a hybrid are the modes involving $\mathrm{S}$-wave ground state meson $+\mathrm{P}$-wave meson. There is also less famous and more model dependent selection rule $[7,8]$ : not only the total angular momentum, but also the total spin of constituents is conserved in the decay process. Application of the selection rules to the ground state hybrid decays leads to the following list of favoured decay modes:

Isovectors:

$1^{--} \rightarrow \pi a_{1}, \rho \varepsilon$

$0^{-+} \rightarrow \pi \varepsilon, \pi f_{0}$

$1^{-+} \rightarrow \pi b_{1}, \pi f_{1}$

$2^{-+} \rightarrow \pi f_{2}$ 
Isoscalars:

$1^{--} \rightarrow \omega \varepsilon, \omega f_{0}$

$0^{-+} \rightarrow \eta \varepsilon, \pi a_{0}$

$1^{-+} \rightarrow \pi a_{1}$

$2^{-+} \rightarrow \pi a_{2}, \eta f_{2}$

An additional advantage exists which can help to tell hybrids from radially excited quarkonia: because of the node in the wave function of radial $q \bar{q}$ excitation the decay of $n S q \bar{q}$ into $\mathrm{S}-$ wave ground state meson $+\mathrm{P}$-wave meson is suppressed. Recently the comprehensive analysis of higher quarkonia decays in the ${ }^{3} P_{0}$ model was performed [9], and it was stressed once more that the studies of the decays into both $\mathrm{S}+\mathrm{S}$ and $\mathrm{S}+\mathrm{P}$ mesons can provide useful information on quarkonium or hybrid assignements. Indeed, the mass estimations are model dependent, and are to be supplied by other considerations, like " $\mathrm{ex}$ tra" states, exotic quantum numbers and so on. The decay signatures play an important role, and the existing hybrid candidates were isolated basing on the analysis of the decay modes mainly.

It is well known that something is wrong with $\rho(1450)$ as the $2 S$ radial $q \bar{q}$ : not only the fourpion mode is dominant, but also the $e^{+} e^{-} \rightarrow$ $\pi^{+} \pi^{-} \pi^{+} \pi^{-}$crossection is several times larger than $e^{+} e^{-} \rightarrow \pi^{+} \pi^{-} \pi^{0} \pi^{0}$ around this resonance [10]. In terms of two - body intermediate states it means that $\pi a_{1}$ is the main decay channel, and $\pi h_{1}$ is remarkably weak, in accordance with expectations for hybrid (spin conservation selection rule). On the other hand, the electromagnetic coupling of a hybrid is much smaller than of $2 S q \bar{q}$, which suggests the mixing scheme [11] to describe $\rho(1450)$.

The $1^{-}\left(0^{-+}\right)$pion with the mass $1.8 \mathrm{GeV}$ was seen in [12], and rediscovered by VES [13]. It decays into $\pi f_{0}$ and $\pi K \bar{K}$, and has a moderate width of about $200 \mathrm{MeV}$, in contrast to expectations for $3 S q \bar{q}$ pion. As the $3 S$ state should be placed somewhre around, careful analysis of other modes, especially $\rho \omega[9]$ is needed to confirm the hybrid component.

The $1^{-+}$channel was always thought of as a hybrid one, because such quantum numbers are unaccessible in the $q \bar{q}$ sector. The resonant activity in $\pi f_{1} 1^{-+}$wave [14] is rather promising; it should be noted, however, that there is another mode, $\pi b_{1}$, with the ratio of branchings $\pi b_{1} / \pi f_{1} \sim 4$ estimated for a hybrid [8]. The observation of $\pi b_{1}$ signal would provide a strong support in favour of hybrid interpretation. It worth mentioning that $\mathrm{BNL}$ has reported the resonances in this wave with much lower masses [15]; if confirmed, this observation might change the adopted hybrid picture in a very funny way.

The tensor channel $2^{-+}$is interesting per se. The $\pi_{2}(1670)$ is a respectable member of $q \bar{q}$ ${ }^{1} D_{2}$ nonet, with strong coupling to $\pi f_{2}$ and $\rho \pi$. There are also evidences for other tensors in the same mass region, with the same decay pattern $[16,17]$. The absence of isoscalar $2^{-+}$states was always intriguing, and, at last, $\eta_{2}$ states begin to appear: the $\eta_{2}$ (1875) from Crystall Ball [18] and $\eta_{2}(1645)$ and $\eta_{2}$ (1875) from Crystall Barrel [19]. As the $q \bar{q}$ in this wave is orbitally excited with no suppression of $\mathrm{S}$-wave $+\mathrm{P}$-wave mesons, the large isovector $\pi f_{2}$ or isoscalar $\pi a_{2}$ modes do not provide decisive arguments for hybrid assignment, and further studies are needed to identify tensor hybrids. Actually, this channel is a perfect playing ground to study the $q \bar{q}-q \bar{q} g$ mixing.

\section{HADRONIC SHIFTS}

The first results of hybrid searches look rather promising, but the life is not as simple as it might seem. The mechanism exists which might change the simple constituent picture drastically, and this mechanism is mixing with hadronic channels and unitarity effects.

The necessity to perform the coupled channel analysis in the formalism which respects unitarity was advocated for long by N.Tornqvist. The unitarity effects can be included via dispersion 
relations approach, and the scalar nonet was recently analysed in such way [20]. The physical mass of the resonance appears to be considerably shifted with respect to the bare constituent model mass due to the coupling to hadronic channels. Correspondingly, the wave function of the physical state contains considerable contribution of hadronic molecule component. The magnitude of effect depends not only on the strength of coupling, but also on how close to the threshold the resomance is, and on the partial wave of hadronic final state. There even might be no oneto-one correspondence between bare state and resonance; the same bare state can manifest itself as relatively narrow structure and profound threshold effect at the same time. Such situation usually occurs if the final hadrons are in relative S-wave, as it takes place for dominant hybrid decays into $\mathrm{S}$-wave $+\mathrm{P}$-wave mesons.

Indeed, the physical mass $m$ of the resonance is defined as the pole of the hadronic $S$-matrix

$m^{2}=m_{0}^{2}+\Delta, \quad \Delta=\sum_{i} \Delta(i)$,

where $m_{0}$ is the bare constituent mass, and the hadronic shift $\Delta$ is the sum over contributions from all hadronic channels $i$. The imaginary part of $\Delta(i)$ is given by decay amplitude of the bare state into channel $i$, and the real part is calculated via dispersion relation:

$\operatorname{Re} \Delta(i)=\int_{s_{i}}^{\infty} d s^{\prime} \frac{\operatorname{Im} \Delta\left(s^{\prime}\right)}{s-s^{\prime}}$.

If the resonance is far from the threshold, one may neglect the dependence on $s$ in the denominator of (8). The approximate sum rule [21] was established for the hadronic shift far from the threshold, which actually means that effect of hadronic loops is simply to renormalize the bare mass.

This sum rule is obviously strongly violated by nearby thresholds, and S-wave thresholds are the strongest ones: the imaginary part $\operatorname{Im} \Delta$ behaves as $k_{i}^{2 l+1}$, and for $l=0$ one has

$\frac{\partial}{\partial s} \operatorname{Re} \Delta(i)=-\int_{s_{i}}^{\infty} d s^{\prime} \frac{\operatorname{Im} \Delta\left(s^{\prime}\right)}{\left(s-s^{\prime}\right)^{2}} \rightarrow \infty$ at the threshold.

Such cusp-like behaviour of the hadronic $S$ matrix might have a drastic effect on observables, obscuring the interpretation of data in terms of bare states. Moreover,the strength of the cusp might be smoothened over some mass interval in the case of hybrid decay into $\mathrm{S}$-wave $+\mathrm{P}$-wave mesons, because the $\mathrm{P}$-wave mesons are usually broad, and it will confuse the picture even more.

\section{CONCLUSIONS}

To summarise, a lot of evidence already exists that the mesons in the mass region $1.5-2.0$ $\mathrm{GeV}$ contain admixture of constituent glue. Still the final conclusions should be done only after performing the coupled channel analysis of existing data.

\section{REFERENCES}

1. G.t'Hooft, Nucl.Phys., B75, (1974) 461

2. S.Dalley, I.R.Klebanov, Phys.Rev., D47 (1993) 2517

3. A.Yu.Dubin, A.B.Kaidalov, Yu.A.Simonov, Phys.Lett., B323 (1994) 41

4. T.Barnes, F.E.Close, E.S.Swanson, Phys. Rev., D52 (1995) 5242

5. Yu.S.Kalashnikova, Yu.B.Yufryakov, Phys. Lett., B359 (1995) 175

6. N.Isgur, R.Kokoski, J.Paton, Phys.Rev.Lett., 54 (1985) 869

F.E.Close, P.R.Page, Nucl.Phys. B443 (1995) 233

7. A.Le Yaonanc, L.Oliver, O.Pene, J.-C.Raynal, Z.Phys. C28 (1985) 309

8. Yu.S.Kalashnikova, Z.Phys., C62 (1994) 323

9. T.Barnes, in these Proceedings T.Barnes, F.E.Close, P.R.Page,E.S.Swanson, MC-TH-96/21, ORNL-CTP-96-09, RAL96-039, hep-ph/9609339

10. A.B.Clegg, A.Donnachie, Z.Phys., C62 (1994) 455

11. A.Donnachie, Yu.S.Kalashnikova, Z.Phys., C59 (1993) 621

12. G.Bellini et.al., Phys.Rev.Lett., 48 (1982) 
1697

13. D.Ryabchikov, in Proceedings of Hadron'95, Manchester, UK, 1994

14. J.H.Lee et.al., Phys.Lett. B323 (1994) 227

S.U.Chung, in these Proceedings

15. S.U.Chung, in these Proceedings

16. G.T.Condo et.al., Phys.Rev., D43 (1991) 2787

17. C.Daum et.al, Nucl.Phys., B182 (1981) 269

18. K.Karch et.al., Z.Phys., C54 (1992) 331

19. D.V.Bugg, in these Proceedings D.V.Bugg et.al., Z.Phys.C, in press

20. N.A.Tornqvist, Z.Phys., C68 (1995) 647

21. P.Geiger, N.Isgur, Phys.Rev., D44 (1991) 799 